\title{
Calculations of switching field and energy barrier for magnetic islands with perpendicular anisotropy
}

\author{
S. Y. Liashko ${ }^{1,2}$, H. Jónsson ${ }^{2,3}$, V. M. Uzdin ${ }^{1,4}$ \\ ${ }^{1}$ ITMO University, Kronverkskiy, 49, St. Petersburg, 197101, Russia \\ ${ }^{2}$ Science Institute and Faculty of Physical Sciences, Univ. of Iceland 107 Reykjavík, Iceland \\ ${ }^{3}$ Center for Nonlinear Studies, Los Alamos, NM 87545, USA \\ ${ }^{4}$ St. Petersburg State University, St. Petersburg, 198504, Russia \\ serjfv@gmail.com, hj@hi.is, v_uzdin@mail.ru
}

DOI 10.17586/2220-8054-2017-8-6-701-708

\begin{abstract}
Calculations of the magnetic field required to reverse the magnetization of islands with out-of-plane anisotropy are carried out using a model describing nucleation followed by rapid domain wall motion. The calculations are based on an extension of the Stoner-Wohlfarth model where thermal activation is taken into account as well as the applied magnetic field. The calculated switching field distribution (SFD) is compared with recently reported experimental measurements of de Vries et al. [New J. Phys. 19, 093019 (2017)] on circular $220 \mathrm{~nm}$ CoPt islands. The measured results can be closely reproduced by choosing appropriate values of two parameters, the nucleation volume, and the effective anisotropy. Both the position of SDF peaks and their width at high and low temperature, $300 \mathrm{~K}$ and $10 \mathrm{~K}$, are amply described using the same set of parameter values for a given island, while there is a large difference between islands with weak and strong magnetic anisotropies. There is no need to introduce the temperature dependence of the activation energy at zero field. This is in contrast with the estimates obtained from the so-called diamond model used by de Vries et al. in their data analysis where multiple adjustable parameters are introduced, and a three- to fourfold change in the zero field activation energy is invoked.
\end{abstract}

Keywords: activation volume, pre-exponential factor, magnetic islands, activation energy, rate theory, spin ice.

Received: 14 November 2017

Revised: 19 November 2017

\section{Introduction}

A large research effort has over the past decades been dedicated to studies of small magnetic particles. Advances in the technology for fabricating such particles of various forms, such as powder samples and ferrofluids, as well as ordered arrangements of magnetic particles have allowed a wide range of interesting experiments. As a result, new physical phenomena have been discovered and technological advances made, such as high anisotropy nanoparticles [1], single-domain magnetic dots [2] and devices for spin manipulation [3]. There is a large potential for such materials in new applications from high-density data storage and logic devices to magnetic sensors. New applications are constantly found, for example in medicine for transportation of chemical substances or transfer of energy to defined target regions in biological systems, both for diagnostics and therapeutics.

One of the most important issues is the description of the magnetization reversal processes in such systems. The bulk magnetic material consists of multiple regions with homogeneous magnetization - domains which are separated by domain walls. The structure and the shape of the domains are strongly dependent on the combination of exchange, magnetostatic and anisotropy energy. If one would decrease the volume of the magnetic system, the size of the domains and the width of the domain walls would also tend to decrease. If the size of the magnetic particles is decreased sufficiently, the formation of the domain wall becomes unfavorable, and only a single magnetic domain is found within the particles [4]. This critical size depends on the saturation magnetization, the exchange energy between the spins and the anisotropy energy. For spherical particles, the critical diameter is on the order of 10-800 $\mathrm{nm}$ [5]. For example, the critical diameters of Fe and Co particles have been estimated to be $15 \mathrm{~nm}$ and $35 \mathrm{~nm}$, respectively, while for $\mathrm{SmCo}_{5}$ an estimate of $750 \mathrm{~nm}$ has been given [6].

Even if the particles have a single magnetic domain, there can be variations in magnetic properties within the particle due to impurities, changes in elemental composition and due to the shape of the particle. As a result, the anisotropy and exchange energy, for example, can vary in different regions of the particle. A reversal of the magnetization in the presence of an applied field can start in regions where the spins can be more easily flipped and then propagate rapidly from there throughout the particle. The field required to reverse the magnetization, the switching field $H_{s w}$, is then strongly affected by imperfections in the particle that lower the activation energy for spin flips. Experiments on continuous [7] as well as patterned [8] magnetic devices including random access 
memory devices [9] have been interpreted successfully in terms of such a nucleation and propagation mechanism for magnetization reversal. The switching field is then largely determined by the volume of the critical nucleus, which in turn determines the activation energy for the magnetization reversal.

Even carefully prepared samples of thin magnetic films on non-magnetic surfaces and magnetic islands patterned from such films have been found to have a wide distribution of nucleation volumes [10-12]. For example, experiments on $\mathrm{Co} / \mathrm{Pd}$ islands as large as $5 \mu \mathrm{m}$ [12] have shown that the switching field distribution (SFD) of patterned islands is a reflection of the spatial variation of the nucleation volume of the continuous film from which the islands were formed. The measurements could be described by a model where the continuous film reverses by nucleation of a low anisotropy volume followed by rapid domain wall propagation. While it might be assumed that high exchange coupling in patterned islands would lead to narrow SFD, compared for example to the thin films, this is not what has been found experimentally $[12,13]$. Experiments, where the angle, $\theta$, between the applied field and the anisotropy axis was varied, showed the well-known $1 / \cos \theta$ dependence for the continuous film, consistent with a mechanism where the reversal rate is limited by wall propagation. However, a minimum in the critical field at $45^{\circ}$ was found for magnetic islands, consistent with the Stoner-Wohlfarth model [14], which describes the rotation of a single magnetic moment of a homogeneous magnetic particle. This indicates that the rotation of the magnetic moment of the critical nucleus can be described by the Stoner-Wohlfarth model.

In recent experiments, de Vries et al. were able to measure the SFDs of individual magnetic islands and found a large variation in the properties of the islands even though the shape and size were the same [15]. In these experiments, the SFD is only a reflection of the stochastic nature of the thermal activation since the measurements are carried out for one island at a time but with repeated sweeps of the magnetic field. The experiments were based on the highly sensitive anomalous Hall effect and were carried out for disk-like islands of $220 \mathrm{~nm}$ diameter patterned from a $\mathrm{Co} / \mathrm{Pt}$ multilayer film. A large difference was found in the maximum field needed to switch the magnetization of different islands, likely due to variations in the thickness and composition of the metallic layers from one island to another. "Weak" islands had, for example, a maximum in the SFD at a field of $84 \mathrm{kA} / \mathrm{m}$ while "strong" islands a maximum at $184 \mathrm{kA} / \mathrm{m}$ in measurements carried out at $10 \mathrm{~K}$ [15]. As the temperature was increased to $300 \mathrm{~K}$, the peaks shifted to the lower field by ca. $50 \mathrm{kA} / \mathrm{m}$ and $30 \mathrm{kA} / \mathrm{m}$ for weak and strong islands, respectively.

These experiments were analyzed by a so-called diamond model, where the shape of the islands is approximated by a square, and the activation energy is related to the length of a domain wall that starts at one corner and then propagates through the island. Several parameters in the model were adjusted in order to reproduce the experimental measurements, including a fairly strong drop in the saturation magnetization, by $7 \%$, and the anisotropy constant, by $16 \%$, as the temperature is increased from $10 \mathrm{~K}$ to $300 \mathrm{~K}$. In earlier research, de Vries et al. concluded from their analysis that the energy barrier for magnetization reversal in zero field is a factor of three to four lower at $300 \mathrm{~K}$ than at $10 \mathrm{~K}$. This is a very large factor and would mean that this fundamental and important parameter could not be obtained from measurements of remagnetization rates over a range in temperature, as is frequently done to establish Arrhenius rate expressions.

In this article, we present calculations using an extended Stoner-Wohlfarth model [16] where the effect of thermal activation has been added and analyze the experimental measurements reported by de Vries et al. An analytical expression for the remagnetization rate, both pre-exponential factor, and activation energy is used to evaluate the SFD for both weak and strong islands. By adjusting two parameters in the model, the effective anisotropy constant and the nucleation volume, to reproduce the measured SFD peak positions, the full shape of the SFD at both $10 \mathrm{~K}$ and $300 \mathrm{~K}$ is found to be in close agreement with the experiments, without the need to vary the values of the parameters with temperature. Contrary to the conclusions described above, we find that a temperature independent energy barrier at zero field can be consistent with the experimental observations of de Vries et al. While the saturation magnetization and anisotropy should, of course, vary to some extent with temperature, our results indicate that it is only a small effect.

In the next section, the model is described including the method for calculating the SFDs. The results on the fitting of the peak positions are then described, followed by a comparison with the full shape of the SFDs. Calculated hysteresis loops for weak and strong islands are presented. Finally, the effect of temperature dependence is illustrated and discussed. The article closes with a conclusion section.

\section{Model}

The calculations are based on an extended Stoner-Wohlfarth model that takes into account thermal fluctuations [16]. This model was recently used to analyze remagnetization in elements of kagome spin ice [17]. In order to mimic the islands measured by de Vries et al., the easy axis is taken to be perpendicular to the surface plane, and the applied magnetic field is directed parallel to the easy axis and opposite to the initial direction of 
the magnetization. A schematic of an island and a critical nucleus for the reversal are shown in Fig. 1 along with the coordinate system used. It is chosen in the same way as in ref. [16] for consistency. In the present case, the magnetic moment entering the model represents a critical nucleus for the remagnetization transition, not the total magnetic moment of the island. It is assumed that the remagnetization transition starts in a small region within the island where for some reason it is easier to reverse the magnetic moments. Once the critical nucleus has formed, the domain of reversed magnetization grows quickly. A key parameter of the model is the volume of the nucleus, $V$, from which the magnetic moment of the nucleus, $M$, can be obtained by multiplying with the saturation magnetization, $M_{s}$, as $M=V M_{s}$. The other key parameter of the model is the anisotropy constant, $K_{1}$. It represents an effective anisotropy that indirectly takes into account interfacial effects between the nucleus and the rest of the island. The experimental measurements of Thomson et al. [12] support the use of such a model to describe remagnetization in magnetic islands that are too large for uniform rotation of all the magnetic moments in the island to be feasible. The parameters $V$ and $K_{1}$ are treated as adjustable model parameters.

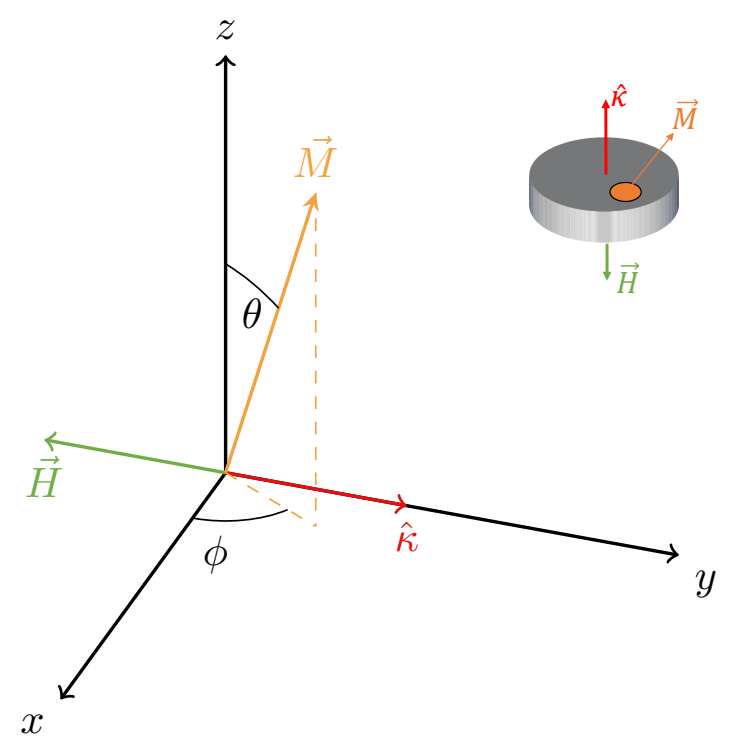

FIG. 1. Schematic representation of a magnetic island and the coordinate system used in the calculations. The out-of-plane anisotropy dominates over the in-plane anisotropy, and the magnetization is pointing along the surface normal, $\hat{\kappa}$. The external magnetic field, $\mathbf{H}$, is directed opposite to the initial state magnetization. The model focuses on the magnetic moment of the critical nucleus of the remagnetization transition, $\mathbf{M}$, pointing in the direction given by $\theta$ and $\phi$.

For an applied magnetic field, $H$, pointing in the direction of the out-of-plane anisotropy axis the extended Stoner-Wohlfarth model gives an analytical expression for the rate of magnetization reversals [16]:

$$
k_{\|}^{H T S T}=f_{0} e^{-\Delta E / k_{B} T}=\frac{\gamma K_{1} \sqrt{(1-h)(2 c-h+1)}}{\pi M_{s}} \exp \left[-\frac{K_{1} V}{k_{B} T}(1-h)^{2}\right],
$$

where $\gamma$ is the gyromagnetic ratio, $h \equiv H M_{0} / 2 K_{1}$, and $c$ is the ratio of the out-of-plane, $K_{1}$, and in-plane, $K_{2}$, anisotropy constants, $c \equiv K_{2} / K_{1}$. The value of $c$ does not affect the calculated results significantly and is simply assumed here to be 0.1 . This expression can be used to estimate the magnetic field needed to reverse the magnetization at a given temperature and for a given timescale. A similar model has been used to analyze the reversal of magnetization in a spring magnet [18].

In typical experimental measurements, including those of de Vries et al., the magnetic field is increased at a constant rate, $R=\Delta H / \Delta t$ until the magnetization reverses. Since the transition is thermally activated, in addition to being aided by the applied field, the field strength at the point when the reversal occurs varies from one measurement to another. Given the pre-exponential factor, $f_{0}$ and activation energy, $\Delta E$, as in the expression for the rate constant above, the probability density for the value of $H$ when the magnetization switches can be 
estimated as [19]:

$$
p_{s w}(H)=\frac{f_{0}(H)}{R} \exp \left(-\frac{\Delta E(H)}{k_{B} T}\right) \times \exp \left[-\frac{1}{R} \int_{-H_{s a t}}^{H^{\prime}} f_{0}\left(H^{\prime}\right) \exp \left(-\frac{\Delta E\left(H^{\prime}\right)}{k_{B} T}\right) d H^{\prime}\right] .
$$

The value of $p_{s w}(H) d H$ gives the probability that the magnetization switching will occur when the field strength is within the interval $[H, H+d H]$ (the first two factors in 2), given the condition that it has not occurred at a smaller value of the field (the last factor in eq 2). This distribution can be compared with a histogram of the switching field in repeated measurements of a single magnetic island, as in the experiments of de Vries et al.. The $M_{s}$ was chosen to have the reported value, $M_{s}=829 \mathrm{kA} / \mathrm{m}$ [15]. The islands where estimated have a diameter of $d=220 \mathrm{~nm}$ and thickness $t=20 \mathrm{~nm}$, giving total volume of $V_{i s l}=760 \cdot 10^{3} \mathrm{~nm}^{3}$, but these dimensions of the islands do not enter the calculations presented here since the focus is on the critical nucleus and its volume rather than the whole island. Only when the ratio of the volume of the nucleus and the total volume of the island is calculated for presentation purposes are the estimated total volume of the islands needed.

Each island is within this model described by the two parameters $K_{1}$ and $V$. Even if all the islands in the array have the same size and round shape, the effective anisotropy and, thereby, nucleation volume can differ significantly, for example, due to variations in the thickness of the metallic layers. Below, a detailed comparison is made between calculations using the model described above and the experimental measurements of de Vries et al. on weak and strong islands at a temperature of $10 \mathrm{~K}$ and $300 \mathrm{~K}$.

\section{Results}

The maxima in the SFD at $10 \mathrm{~K}$ and at $300 \mathrm{~K}, H_{10 \mathrm{~K}}^{m}$ and $H_{300 \mathrm{~K}}^{m}$, were calculated over a range of values for the two model parameters, $K_{1}$ and $V$. The results are shown in Fig. 2. The contour graph shows the value of the difference, $\Delta H^{m}=H_{10 K}^{m}-H_{300 K}^{m}$, and the two colored curves show the values of $K_{1}$ and $V$ that give $H_{10 K}^{m}$ in agreement with the values obtained by de Vries et al. for weak and strong islands. The measured shift, $\Delta H^{m}$, is ca. $50 \mathrm{kA} / \mathrm{m}$ for weak islands and $30 \mathrm{kA} / \mathrm{m}$ for strong islands. The low-temperature peaks, $H_{10}^{m}$, are largely determined by $K_{1}$ but $V$ affects the calculated temperature dependence of the peak positions.

For one particular set of parameters, $K_{1}$ and $V$, the measured values of both $H_{10 K}^{m}$ and $H_{300 K}^{m}$ can be reproduced for each type of island. The peak in the SFD at $10 \mathrm{~K}$ is largely determined by the anisotropy parameter and is weakly dependent on the nucleation volume (thus the small slope of the colored lines). The temperature shift, however, depends also on $\mathrm{V}$ and the isolines of constant temperature shift in $H^{m}$ are nearly straight lines with a significant slope.

Figure 3 shows the measured and calculated SFDs. It is evident from the figure that close agreement between the calculated and measured peak positions can be obtained by adjusting the two parameters. The optimal values are $K_{1}=99.28 \mathrm{~kJ} / \mathrm{m}^{3}$ and $V=0.036 V_{i s l}$ for strong islands, and $K_{1}=49.46 \mathrm{~kJ} / \mathrm{m}^{3}$ and $V=0.0067 V_{i s l}$ for weak islands.

Not only are the measured peak positions reproduced well, but also the shape of the calculated SDFs is in agreement with the measurements of de Vries et al. [15]. Since the width of the SFD peaks is not part of the fitting procedure, this lends strong support for the appropriateness of the present model for this system. Also, it is interesting that this close agreement with the experimental data can be obtained with the same values of $K_{1}$ and $V$ over such widely different temperature values, $10 \mathrm{~K}$ and $300 \mathrm{~K}$. The shift in peak position and the increased width of the peaks as temperature is increased is accurately described by harmonic transition state theory for magnetic systems [20] on which the extended Stoner-Wohlfarth model is based.

The zero field activation energy obtained from the model using these values of the parameters is $1.6 \mathrm{eV}$ for weak and $17.0 \mathrm{eV}$ for strong islands. The difference is indeed very large, showing that the inhomogeneity is strong. The calculated pre-exponential factors in the rate expression, eqn. (1), at zero field are $3.5 \cdot 10^{9} \mathrm{~Hz}$ and for weak and $7.1 \cdot 10^{9} \mathrm{~Hz}$ for strong islands. These values are close to what has been assumed for Arrhenius rate expressions in the previous analysis [15,21]. However, in the present approach, these values are obtained from the extended Stoner-Wohlfarth model.

Calculated hysteresis loops for weak and strong islands are shown in Fig. 4. The SFD is used there to produce loops for each type of island representing averages obtained in repeated scans of the applied field. The narrowing of the loops as the temperature is increased from $10 \mathrm{~K}$ to $300 \mathrm{~K}$ is clearly seen as well as the rounding of the corners due to the stochastic nature of the thermal activation. While the applied field lowers the activation energy the system needs to overcome to reverse the magnetization, the transitions eventually occur due to thermal fluctuations. So, different values of the critical field are obtained in different scans of the field strength. 


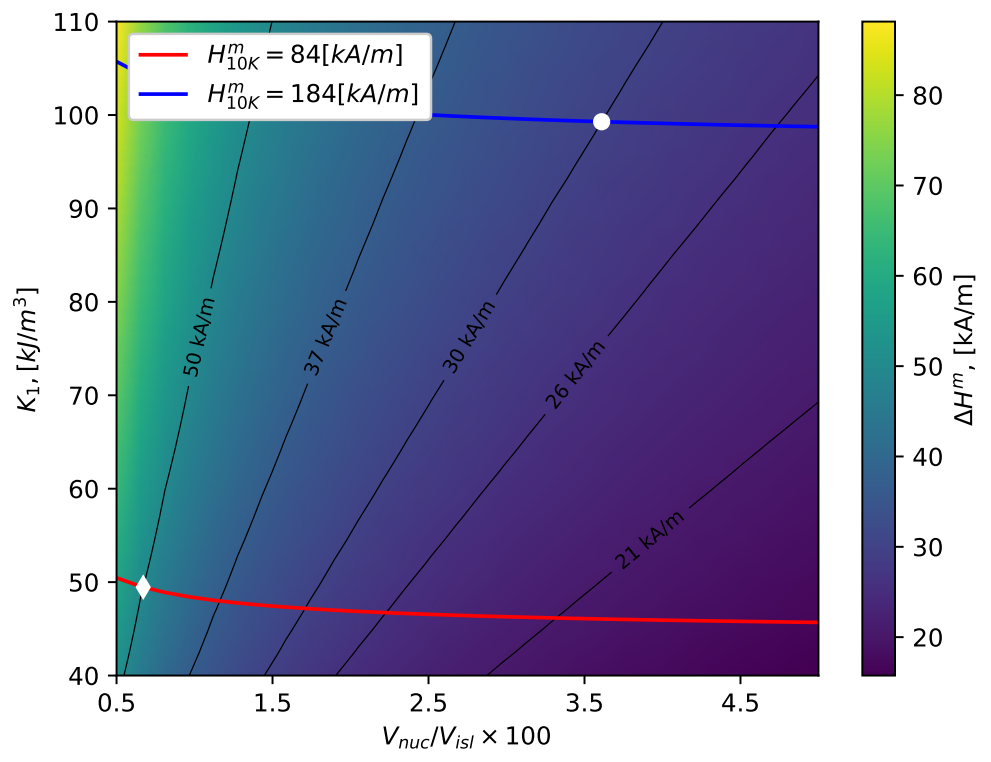

FIG. 2. Contour graph of the calculated shift in SFD peak positions when the temperature is increased from $10 \mathrm{~K}$ to $300 \mathrm{~K}$, i.e., $\Delta H^{m}=H_{10 K}^{m}-H_{300 K}^{m}$, as a function of the two adjustable parameters, the anisotropy constant, $K_{1}$, and the nucleation volume, $V$ (given as the fraction of the total volume $V_{i s l}=760 \cdot 10^{3} \mathrm{~nm}^{3}$ ). Parameter values that give peak positions at $10 \mathrm{~K}$, i.e., $H_{10 K}^{m}$ in agreement with experimental observations [15] are shown with lines (red for a weak island, blue for a strong island). White diamond and disk identify parameter values that give peak positions in agreement with experiments at both $10 \mathrm{~K}$ and $300 \mathrm{~K}$ for weak and strong islands, respectively. The figure illustrates how a unique set of the two adjustable parameters can be found in such a way as to obtain agreement between the calculated and measured position of maxima in the SFD.
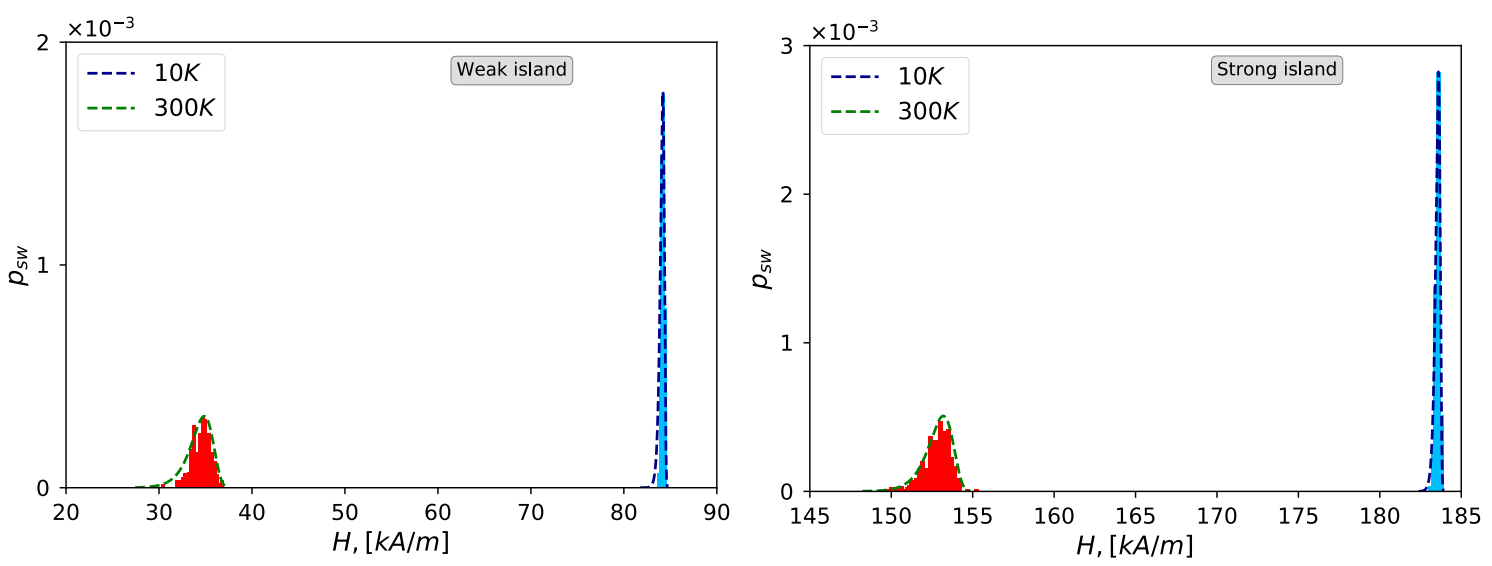

FIG. 3. Comparison of calculated (dashed lines) and measured (histograms) [15] SFDs for weak and strong islands at $10 \mathrm{~K}$ and at $300 \mathrm{~K}$. The values of the two parameters, $K_{1}$ and $V$, in the theoretical model were obtained by adjusting the positions of the maxima of the SFD (see Fig. 2), but the shape of the SDFs then turns out to also be in close agreement with the experimental measurements. 

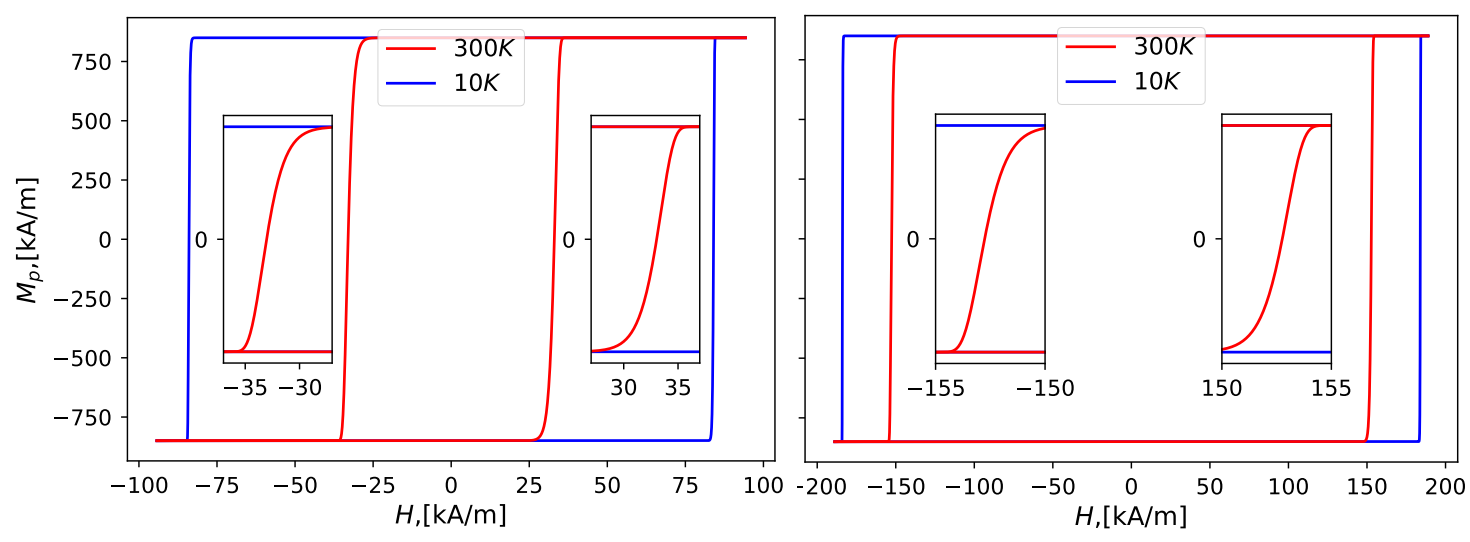

FIG. 4. Calculated hysteresis for a weak (left) and strong (right) island obtained from the calculated SFDs. The insets show on an expanded scale the rounding of the corners due to the stochastic nature of the thermal activation.

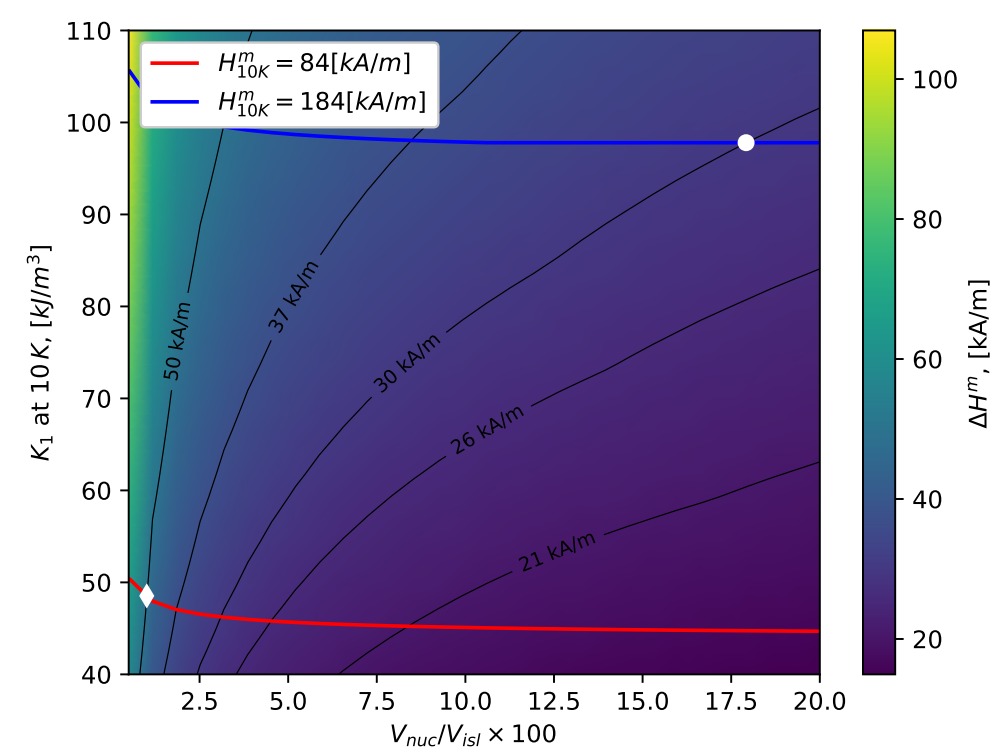

FIG. 5. Contour graph similar to Fig. 2 except here the saturation magnetization, $\mathrm{M}_{s}$, is assumed to drop by $7 \%$ and the anisotropy constant, $K_{1}$, by $16 \%$ as the temperature is increased from $10 \mathrm{~K}$ to $300 \mathrm{~K}$, as estimated by de Vries et al. [15]. Parameter values that give peak positions at $10 \mathrm{~K}$, i.e., $H_{10 \mathrm{~K}}^{m}$ in agreement with experimental observations [15] are shown with lines (red for a weak island, blue for a strong island). The value of the nucleation volume, $V$, needed to reproduce the shift in SDF peak position as the temperature is increased from $10 \mathrm{~K}$ to $300 \mathrm{~K}$ is much larger and the contour lines are more curved than when the parameters are temperature independent (see Fig. 2).

It is interesting that such close agreement with the experimental data can be obtained without changing the values of $K_{1}$ and $V$ as well as $M_{s}$ at the two values for the temperature, $10 \mathrm{~K}$ to $300 \mathrm{~K}$. There will, of course, be some temperature dependence of these parameters, but the model used here indicates that it should be small. Previous estimates by de Vries et al. from their analysis showed a decrease in $M_{s}$ by about $7 \%$ and a decrease in $K_{1}$ by about $16 \%$ as the temperature is increased from $10 \mathrm{~K}$ to $300 \mathrm{~K}$ [15]. It is indeed possible to reproduce the positions of the peaks in the SFDs with such a strong temperature dependence of these parameter values, but the nucleation volume, $V$, then needs to become 4 to 5 times larger at $300 \mathrm{~K}$ than at $10 \mathrm{~K}$. As a result, the width of the SFD becomes much too narrow, by more than an order of magnitude, in strong disagreement with the experimental measurements. Within the present model, the decrease in the values of $K_{1}$ and $V$ as well as $M_{s}$ must be much smaller than the estimate of de Vries et al. in order to reproduce the experimental observations. 


\section{Conclusion}

A simple model based on an extended Stoner-Wohlfarth equation for magnetization reversal in an island where the transition mechanism involves nucleation followed by rapid domain wall motion is found to reproduce well the elegant measurements of de Vries et al. [15] of SFDs of individual magnetic islands. The two adjustable parameters in the model, the anisotropy constant and the nucleation volume can be determined from the SFD peak positions at $10 \mathrm{~K}$ and $300 \mathrm{~K}$. It is not necessary to include temperature dependence of the parameters. The shape of the SFD is then also found to be in close agreement with the measurements. The conclusion from this analysis of the experimental data is that the activation energy in zero field can be taken to be independent of temperature. This is in contradiction to the conclusion of de Vries et al. based on the diamond model, where the zero field activation energy is decreased by a factor of three to four (for weak vs. strong islands) as the temperature is increased from $10 \mathrm{~K}$ to $300 \mathrm{~K}$. This represents a large and important difference in the analysis of the experimental data. If indeed the zero field activation energy were to change so much with temperature, the standard methodology for determining activation energy from measurements taken over a wide temperature interval could not be applied when determining this important quantity. Instead, our analysis indicates that the standard approach is applicable and can give a proper characterization of the magnetization reversal transition.

Our analysis does not assume the value of the pre-exponential factor, $f_{0}$, in the rate expression, eqn. (1). Rather, it is obtained from the model. While the magnitude of the pre-exponential factor turns out to be between $10^{9} \mathrm{~Hz}$ and $10^{10} \mathrm{~Hz}$ in the present case, similar to what is often assumed for magnetic transitions, it is important to realize that harmonic transitions state theory has in some cases been found to give quite different values for pre-exponential factors in magnetic reversals (see, for example, [22,23]). In general, it is important to evaluate the pre-exponential factor rather than just to assume some value.

\section{Acknowledgements}

This work was supported by the Icelandic Research Fund, the Academy of Finland (grant 278260) and the Government of the Russian Federation (grant 074U01).

\section{References}

[1] Sun S., Murray C.B., Weller D., Folks L., Moser A. Monodisperse FePt nanoparticles and ferromagnetic FePt nanocrystal superlattices. Science, 2000, 287(5460), P. 1989-1992.

[2] Allwood D.A., Xiong G., Faulkner C.C., Atkinson D., Petit D., Cowburn R.P. Magnetic domain-wall logic. Science, 2005, 309(5741), P. 1688-1692.

[3] Katine J.A., Albert F.J., Buhrman R.A., Myers E.B., Ralph D.C. Current-driven magnetization reversal and spin-wave excitations in $\mathrm{Co} / \mathrm{Cu} / \mathrm{Co}$ pillars. Physical Review Letters, 2000, 84(14), P. 3149.

[4] Jacobs S., Bean C.P. Fine Particles, Thin Films and Exchange Anisotropy. Magnetism vol. III, ed. Rado G.T. and Suhl H. (New York: Academic), 1963, P. 275.

[5] Zijlstra H. Permanent magnets, theory. Handbook of Magnetic Materials, vol. 3, ed. Wohlfarth E.P. (Amsterdam: North-Holland), 1982, P. 37-107.

[6] Givord D., Lu Q., Rossignol M.F. Coercivity in hard magnetic materials. In Science and Technology of Nanostructured Magnetic Materials, Springer US, 1991, P. 635-656.

[7] Zhu J.G., Peng Y., Laughlin D.E. Toward an understanding of grain-to-grain anisotropy field variation in thin film media. IEEE transactions on magnetics, 2005, 41(2), P. 543-548.

[8] Terris B.D., Thomson T. Nanofabricated and self-assembled magnetic structures as data storage media. Journal of physics D: Applied physics, 2005, 38(12), P. R199.

[9] Engel B.N., Akerman J., Butcher B., Dave R.W., DeHerrera M., Durlam M., Slaughter J.M., et al. A 4-Mb toggle MRAM based on a novel bit and switching method. IEEE Transactions on Magnetics, 2005, 41(1), P. 132-136.

[10] Jang H.J., Eames P., Dahlberg E.D., Farhoud M., Ross C.A. Magnetostatic interactions of single-domain nanopillars in quasistatic magnetization states. Applied Physics Letters, 2005, 86(2), P. 023102.

[11] Kitade Y., Komoriya H., Maruyama T. Patterned media fabricated by lithography and argon-ion milling. IEEE transactions on magnetics, 2004, 40(4), P. 2516-2518.

[12] Thomson T., Hu G., Terris B.D. Intrinsic Distribution of Magnetic Anisotropy in Thin Films Probed by Patterned Nanostructures. Phys. Rev. Lett., 2006, 96, P. 257204.

[13] Shaw J.M., Rippard W.H., Russek S.E., Reith T., Falco C.M. Origins of switching field distributions in perpendicular magnetic nanodot arrays. Journal of Applied Physics, 2007, 101(2), P. 023909.

[14] Stoner E.C., Wohlfarth E.P. A mechanism of magnetic hysteresis in heterogeneous alloys. Phil. Trans. Roy. Soc. A, 1948, 240, P. 599-642.

[15] de Vries J., Bolhuis T., Abelmann L. Temperature dependence of the energy barrier and switching field of sub-micron magnetic islands with perpendicular anisotropy. New J. Phys., 2017, 19, P. 093019.

[16] Liashko S.Y., Uzdin V.M., Jónsson H. Thermal stability of magnetic states in submicron magnetic islands. Nanosystems: Physics, Chemistry, Mathematics, 2017, 8(5), P. 572-578.

[17] Liashko S.Y., Jónsson H., Uzdin V.M. The effect of temperature and external field on transitions in elements of kagome spin ice. New J. Phys., 2017, 19, P. 113008. 
[18] Moskalenko M., Bessarab P.F., Uzdin V.M., Jónsson H. Qualitative Insight and Quantitative Analysis of the Effect of Temperature on the Coercivity of a Magnetic System. AIP Advances, 2016, 6(2), P. 025213.

[19] Engelen J.B., Delalande M., Le Febre A.J., Bolhuis T., Shimatsu T., Kikuchi N., Abelmann L., Lodder J.C. Thermally induced switching field distribution of a single CoPt dot in a large array. Nanotechnology, 2009, 21(3), P. 035703.

[20] Bessarab P.F., Uzdin V. M. Jónsson H. Harmonic Transition State Theory of Thermal Spin Transitions. Phys. Rev. B, 2012, 85(18), P. 184409.

[21] Engelen J.B.C., Delalande M., le Febre A.J., Bolhuis T., Shimatsu T., Kikuchi N., Abelmann L., Lodder J.C. Thermally induced switching field distribution of a single CoPt dot in a large array. Nanotechnology, 2010, 21(3), P. 035703.

[22] Bessarab P.F., Uzdin V.M. Jónsson H. Size and Shape Dependence of Thermal Spin Transitions in Nanoislands. Phys. Rev. Lett., 2013, 110(2), P. 020604.

[23] Ivanov A.A., Bessarab P.F., Uzdin V.M., Jónsson H. Magnetic exchange force microscopy: Theoretical analysis of induced magnetization reversals. Nanoscale, 2017, 9(35), P. 13320-13325. 